\title{
Caracterización de mezclas semidensas con agregados de la región de Tunja
}

\author{
Characterization of the Gap Graded Asphalt Mixtures with some \\ Aggregates from the Tunja's City Region
}

Fecha de Recepción: 12 de Diciembre de 2013

Fecha de Aprobación: 06 de Enero de 2014
Carlos Hernando Higuera-Sandoval ${ }^{*}$ Jeiny Elizabeth Aranda-López** José Alberto Prieto-Hernández

\section{Resumen}

Esta investigación determinó la viabilidad de las Mezclas Semidensas en Caliente, o Gap Graded, para el uso en vías de tránsito medio, como una alternativa a la capa asfáltica y a la base asfáltica. Para elaborar las mezclas se utilizaron agregados de dos canteras de la Provincia Centro del departamento de Boyacá: la recebera Piedra Gorda y la cantera La Calera, junto con el ligante bituminoso de penetración 60/70, proveniente de IncoAsfaltos S.A., empresa localizada en Mosquera (Cundinamarca). El diseño de las mezclas se realizó por medio de las metodologías Marshall y Ramcodes. La determinación de los módulos dinámicos de las mezclas se llevó a cabo por medio del equipo Nottingham Asphalt Tester (NAT). Los resultados mostraron valores de módulos mayores para las mezclas realizadas con el agregado de Piedra Gorda. Se diseñó la

\section{Abstract}

The hot Gap graded asphalt mixtures developed in this research, determined their viability to be used in the secondary road transit routes, as an alternative to the asphalt binder and the asphalt base. The aggregates came from two quarries of the Boyaca's Department Central Province, the Recebera Piedra Gorda and La Calera; together with the penetration 60/70's bituminous binder, from IncoAsfaltos S.A., asphalt plant, located in Mosquera (Cundinamarca).

For the mix design, were used the Marshall and Ramcodes methodologies. The mixtures dynamic module's determination was carried out by a Nottingham Asphalt Tester (NAT)'s equipment. The results showed higher modules values for the mixtures made with Piedra Gorda aggregates. The pavement structure was designed with La Calera aggregates, in order to meet all specifications

\footnotetext{
* M.Sc. Universidad Pedagógica y Tecnológica de Colombia (Tunja-Boyacá, Colombia). carlos.higuera@uptc.edu.co

** Universidad Pedagógica y Tecnológica de Colombia (Tunja-Boyacá, Colombia). jeiny.aranda@uptc.edu.co

*** Universidad Pedagógica y Tecnológica de Colombia (Tunja-Boyacá, Colombia). jose.prieto@uptc.edu.co
} 
Caracterización de mezclas semidensas con agregados de la región de Tunja

estructura de pavimento con agregados de $\mathrm{La}$ Calera, por cumplir con todas las especificaciones.

Palabras clave: Agregados pétreos, Asfalto 60/70, Mezcla semidensa en caliente, Capa asfáltica, Base asfáltica.
Keywords: Rocky aggregates, Asphalt 60/70, Hot Gap Graded Mixtures, Asphalt Binder, Asphalt Base. 


\section{INTRODUCCIÓN}

Las condiciones actuales de la infraestructura vial en el país [1], tal como los malos diseños y la baja calidad de los pavimentos, han contribuido a que el desarrollo de la nación y la competitividad económica [2] no progresen, dado que la mayor cantidad de mercancías se transportan por las vías nacionales y, por ende, existe un alto flujo de vehículos de carga que generan mayores daños y repercusiones negativas a las estructuras viales.

En los últimos meses, el gobierno nacional ha decidido buscar alternativas que lleven a una mejora de las condiciones de conectividad entre los centros de producción y los puertos marítimos nacionales; para ello ha destinado una cantidad considerable de recursos económicos que se invertirán en los estudios preliminares, el diseño y la construcción de nuevas vías, que van desde las vías terciarias, para la conexión municipal, hasta las vías nacionales, de doble calzada, que permitan una circulación vehicular más rápida y eficiente, con menores costos de operación para los usuarios.

La construcción de la nueva infraestructura vial de Colombia empieza a generar confianza inversionista, tanto nacional como extranjera, así como necesidad de buscar recursos humanos para los trabajos de ingeniería y materiales que satisfagan las especificaciones de construcción, para su uso en cualquiera de las capas del pavimento.

Basados en las especificaciones del Instituto Nacional de Vías (INVÍAS, 2007) y del Instituto de Desarrollo Urbano (IDU, 2005) para un nivel de tránsito 2 (NT2, según INVÍAS 2007) [3] y nivel de tránsito 5 (T5, según IDU 2005) [4], se fabricaron mezclas semidensas en caliente, cuya característica principal se basa en el porcentaje de vacíos con aire presente en las mezclas elaboradas.
Con el desarrollo de esta investigación se pretende establecer las características físicas de los agregados de dos canteras de la Provincia Centro del departamento de Boyacá, y de asfalto de penetración 60/70; estas mezclas fueron analizadas mediante la implementación de las metodologías Marshall y Ramcodes. Una vez determinado el contenido óptimo de asfalto, se realizaron las mezclas para la aplicación del ensayo de tracción indirecta, haciendo uso del equipo NAT -Nottingham Asphalt Tester-.

\section{DESCRIPCIÓN DEL ESTUDIO EXPERIMENTAL}

\section{A. Caracterización de materiales}

En las mezclas asfálticas, los dos principales materiales son el agregado pétreo y el asfalto; debido a que, dependiendo del lugar de extracción del material, sus características propias pueden variar, es necesario realizar ensayos que permitan identificarlas. Las mezclas asfálticas semidensas en caliente son mezclas homogéneas y uniformes cuya constitución granulométrica tiene un fuerte esqueleto mineral; es por esto que se realizaron ensayos de caracterización de agregados pétreos provenientes de dos canteras ubicadas en la provincia Centro del departamento de Boyacá, y de asfalto de penetración 60/70. La primera fuente de material es la recebera Piedra Gorda, localizada en el Km. 6 de la vía que de Tunja conduce a Villa de Leyva; la extracción del material apto para la construcción de obras civiles se hace a cielo abierto, y la trituración del material se da por medios mecánicos. La segunda fuente de agregado pétreo es la cantera La Calera, ubicada $2 \mathrm{~km}$. al noroeste del municipio de Sora; esta cantera produce material que se utiliza para mezclas asfálticas, mezclas hidráulicas y, en general, todo tipo de obra civil; su trituración y selección se hace mediante equipos mecánicos que categorizan los agregados en función del tamaño y de sus características. En la Tabla 1 se presentan 
los resultados obtenidos en la caracterización del agregado pétreo.

Las mezclas fueron realizadas de acuerdo con la franja granulométrica central planteada por el Instituto Nacional de Vías, INVÍAS-07, y por el Instituto de Desarrollo Urbano, IDU-05. Debido a que en la Uptc no se habían realizado este tipo de mezclas, se presentan en las Tablas 2 y 3 los límites de las franjas granulométricas para las MSC 1 ó MS 20, que servirán como referencia para su uso en las bases asfálticas, y las MSC 2 ó MS 12, que estarán en servicio en las capas de rodadura.

TABLA 1

CARACTERIZACIÓN DEL AGREGADO PÉTREO

\begin{tabular}{|c|c|c|c|c|c|c|c|c|}
\hline \multicolumn{2}{|l|}{ Propiedad } & Norma & $\begin{array}{c}\text { Valor } \\
\text { norma } \\
\text { INVÍAS }\end{array}$ & $\begin{array}{c}\text { Valor } \\
\text { norma } \\
\text { IDU }\end{array}$ & \multicolumn{2}{|c|}{$\begin{array}{c}\text { Resultados } \\
\text { recebera } \\
\text { Piedra Gorda }\end{array}$} & \multicolumn{2}{|c|}{$\begin{array}{l}\text { Resultados cantera } \\
\text { La Calera }\end{array}$} \\
\hline \multirow{3}{*}{ Desgaste de los ángeles } & & \multirow[b]{2}{*}{ INV E 218-07 } & $25 \%$ & $25 \%$ & \multirow{3}{*}{\multicolumn{2}{|c|}{$24.30 \%$}} & \multirow{3}{*}{\multicolumn{2}{|c|}{$20.24 \%$}} \\
\hline & Rodadura & & máx. & máx. & & & & \\
\hline & Base & IDU 510-05 & máx. & máx. & & & & \\
\hline \multirow{2}{*}{\multicolumn{2}{|c|}{$\begin{array}{l}\text { Pérdidas en ensayo de solidez (Sulfato } \\
\text { de sodio) }\end{array}$}} & INV E 220-07 & $12 \%$ & No aplica & Fino & $7.20 \%$ & Fino & $4.59 \%$ \\
\hline & & IDU 510-05 & máx. & Grueso & $3.37 \%$ & Grueso & $1.40 \%$ & \\
\hline \multirow{3}{*}{$\begin{array}{c}\text { Partículas fracturadas } 2 \\
\text { caras }\end{array}$} & & \multirow{3}{*}{$\begin{array}{l}\text { INV E 227-07 } \\
\text { IDU 510-05 }\end{array}$} & $60 \%$ & \multirow{3}{*}{$75 \%$} & \multirow{3}{*}{\multicolumn{2}{|c|}{$88.45 \%$}} & \multirow{3}{*}{\multicolumn{2}{|c|}{$97.08 \%$}} \\
\hline & Rodadura & & mín. & & & & & \\
\hline & Base & & $\begin{array}{l}60 \% \\
\min \\
\end{array}$ & & & & & \\
\hline \multicolumn{2}{|c|}{ Índice de alargamiento } & INV E 230-07 & $35 \%$ & $20 \%$ & \multicolumn{2}{|c|}{$19.48 \%$} & \multicolumn{2}{|c|}{$18.29 \%$} \\
\hline \multicolumn{2}{|c|}{ Índice de aplanamiento } & $\begin{array}{l}\text { INV E 230-07 } \\
\text { IDU 510-05 }\end{array}$ & $\begin{array}{l}35 \% \\
\text { máx. }\end{array}$ & $\begin{array}{l}20 \% \\
\text { máx. }\end{array}$ & \multicolumn{2}{|c|}{$18.83 \%$} & \multicolumn{2}{|c|}{$16.56 \%$} \\
\hline \multirow{4}{*}{\multicolumn{2}{|c|}{$\begin{array}{c}\text { Gravedad específica y absorción de los } \\
\text { agregados finos }\end{array}$}} & \multirow{4}{*}{ INV E 222-07 } & & \multirow{4}{*}{$\begin{array}{l}\text { Gsb sss } \\
\text { Gsa } \\
\% \mathrm{Abs}\end{array}$} & Gsb & 2.593 & Gsb & 2.602 \\
\hline & & & & & 2.612 & Gsb sss & 2.629 & \\
\hline & & & & & 2.643 & Gsa & 2.675 & \\
\hline & & & & & $8.27 \%$ & $\%$ Abs & $1.04 \%$ & \\
\hline \multirow{4}{*}{\multicolumn{2}{|c|}{$\begin{array}{c}\text { Gravedad específica y absorción de los } \\
\text { agregados gruesos }\end{array}$}} & \multirow{4}{*}{ INV E 223-07 } & & Gsb sss & Gsb & 2.502 & Gsb & 2.481 \\
\hline & & & & \multirow[b]{3}{*}{$\% \mathrm{Abs}$} & 2.521 & Gsb sss & 2.530 & \\
\hline & & & & & 2.551 & Gsa & 2.610 & \\
\hline & & & & & $0.75 \%$ & $\% \mathrm{Abs}$ & $1.99 \%$ & \\
\hline
\end{tabular}




\begin{tabular}{|c|c|c|c|c|c|}
\hline Propiedad & Norma & $\begin{array}{c}\text { Valor } \\
\text { norma } \\
\text { INVÍAS }\end{array}$ & $\begin{array}{c}\text { Valor } \\
\text { norma } \\
\text { IDU }\end{array}$ & $\begin{array}{c}\text { Resultados } \\
\text { recebera } \\
\text { Piedra Gorda }\end{array}$ & $\begin{array}{c}\text { Resultados cantera } \\
\text { La Calera }\end{array}$ \\
\hline Límite líquido & $\begin{array}{l}\text { INV E 125-07 } \\
\text { IDU 510-05 }\end{array}$ & N.P. & N.P. & & N.P. \\
\hline Límite plástico & $\begin{array}{l}\text { INV E 126-07 } \\
\text { IDU 510-05 }\end{array}$ & N.P. & N.P. & N.P. & N.P. \\
\hline Índice plástico & $\begin{array}{l}\text { INV E 126-07 } \\
\text { IDU 510-05 }\end{array}$ & N.P. & N.P. & N.P. & N.P. \\
\hline Equivalente de arena & $\begin{array}{l}\text { INV E 133-07 } \\
\text { IDU 510-05 }\end{array}$ & $\begin{array}{l}50 \% \\
\min \end{array}$ & $\begin{array}{l}40 \% \\
\text { mín. }\end{array}$ & $63 \%$ & $76 \%$ \\
\hline
\end{tabular}

TABLA 2

FRANJAS GRANULOMÉTRICAS PARA LAS MSC SEGÚN EL INVÍAS- 07

\begin{tabular}{|c|c|c|c|c|c|c|c|c|c|c|}
\hline \multirow{2}{*}{$\begin{array}{l}\text { TIPO DE } \\
\text { MEZCLA }\end{array}$} & \multicolumn{10}{|c|}{ TAMIZ } \\
\hline & $1 \frac{1}{2}$, & $1 "$ & $3 / 4 "$ & 1/2" & $3 / 8$ & N. ${ }^{\circ} 4$ & N..$^{\circ} 10$ & N..$^{\circ} 40$ & N. ${ }^{\circ} 80$ & N. ${ }^{\circ} 200$ \\
\hline MSC 1 & & 100 & $80-95$ & $65-80$ & $55-70$ & $40-55$ & $24-38$ & $9-20$ & $6-12$ & $3-7$ \\
\hline MSC 2 & & & 100 & $80-95$ & $65-80$ & $40-55$ & $24-38$ & $9-20$ & $6-12$ & $3-7$ \\
\hline
\end{tabular}

TABLA 3

FRANJAS GRANULOMÉTRICAS PARA LAS MSC SEGÚN EL IDU-05

\begin{tabular}{|c|c|c|c|c|c|c|c|c|c|c|}
\hline \multirow{2}{*}{$\begin{array}{l}\text { TIPO DE } \\
\text { MEZCLA }\end{array}$} & \multicolumn{10}{|c|}{ TAMIZ } \\
\hline & $11 / 2 "$ & $1 "$ & $3 / 4 "$ & 1/2" & 3/8" & N. ${ }^{\circ} 4$ & $\mathrm{~N}^{\circ} .^{\circ} 10$ & N. ${ }^{\circ} 40$ & N. ${ }^{\circ} 80$ & N..$^{\circ} 200$ \\
\hline MS - 12 & & & 100 & $80-95$ & $67-83$ & $40-56$ & $23-39$ & $10-20$ & $6-13$ & $3-8$ \\
\hline MS - 20 & & 100 & $80-95$ & $66-82$ & $55-71$ & $35-51$ & $23-39$ & $10-20$ & $6-13$ & $3-8$ \\
\hline
\end{tabular}

En la Tabla 4 se presentan las principales características de este tipo de asfalto, la temperatura de mezcla y de compactación. 
TABLA 4

RESULTADOS DE LOS ENSAYOS REALIZADOS AL ASFALTO DE PENETRACIÓN 60/70

\begin{tabular}{|c|c|c|c|c|c|c|}
\hline \multirow[t]{2}{*}{ ENSAYO } & \multirow[t]{2}{*}{ NORMA } & \multicolumn{2}{|c|}{$\begin{array}{l}\text { VALOR } \\
\text { NORMA } \\
\text { INVÍAS }\end{array}$} & \multicolumn{2}{|c|}{$\begin{array}{c}\text { VALOR } \\
\text { NORMA } \\
\text { IDU } \\
\end{array}$} & \multirow[t]{2}{*}{$\begin{array}{l}\text { INCOASFALTOS } \\
\text { S.A. }\end{array}$} \\
\hline & & Min. & Máx. & Min. & Máx. & \\
\hline $\begin{array}{l}\text { Penetración } \\
\text { (1/10 mm) }\end{array}$ & $\begin{array}{l}\text { INV E 706-07 } \\
\text { IDU 200-05 }\end{array}$ & 60 & 70 & 60 & 70 & 66.3 \\
\hline Índice de penetración & $\begin{array}{l}\text { INV E 724-07 } \\
\text { IDU 200-05 }\end{array}$ & & & -1.5 & 1.5 & -0.6 \\
\hline $\begin{array}{l}\text { Punto de ablandamiento (Método } \\
\text { anillo y bola) }{ }^{\circ} \mathrm{C}\end{array}$ & $\begin{array}{l}\text { INV E } 71207 \\
\text { IDU 200-05 }\end{array}$ & 45 & 55 & 45 & 55 & $49.5^{\circ} \mathrm{C}$ \\
\hline Peso específico & $\begin{array}{l}\text { INV E } 70707 \\
\text { IDU 200-05 }\end{array}$ & & & & & 1.019 \\
\hline $\begin{array}{l}\text { Punto de ignición (copa abierta de } \\
\text { Cleveland) } \mathrm{C}^{\circ}\end{array}$ & $\begin{array}{l}\text { INV E } 70907 \\
\text { IDU 200-05 }\end{array}$ & 230 & - & 232 & - & $282^{\circ} \mathrm{C}$ \\
\hline $\begin{array}{c}\text { Viscosidad a } 60^{\circ} \mathrm{C} \\
\text { (P) }\end{array}$ & $\begin{array}{l}\text { INV E } 71707 \\
\text { IDU 200-05 }\end{array}$ & 1500 & - & 1500 & 3000 & $2648.9 \mathrm{P}$ \\
\hline \multicolumn{6}{|c|}{ Temperatura de mezcla } & $145-150^{\circ} \mathrm{C}$ \\
\hline Tempe & itura de compac & ción & & & & $138-141^{\circ} \mathrm{C}$ \\
\hline
\end{tabular}

\section{B. Metodologías implementadas}

En el desarrollo de la investigación se implementaron las metodologías Marshall y Ramcodes. La metodología Marshall se basa en tres parámetros: a) gravedad específica, b) análisis de densidad y vacíos y c) estabilidad y flujo, con el fin de obtener el contenido óptimo de asfalto y el porcentaje de agregado requerido para la mezcla. La metodología Ramcodes (Metodología Racional para el análisis de densificación y resistencia de geomateriales compactados) se basa en el diseño experimental de dos factores: el contenido de asfalto $(\% \mathrm{~Pb})$ y el peso específico Bulk (Gmb), mediante la representación de un polígono de vacíos, que es demarcado por isolíneas que representan los rangos exigidos en las especificaciones [5].
Con el uso de las ecuaciones (1), (2) y (3) se determinan las isolíneas que definen el polígono de vacíos:

$G m b=\left[\frac{1-V a}{\frac{P b}{G b}+\frac{(1-P b)}{G s e}}\right]$

$G m b=\left[\frac{1-V A M}{1-P b}\right] * G s b(2)$

$G m b=\left[\frac{V F A}{\frac{P b}{G b}+\frac{(1-P b)}{G s e}-(1-V F A) * \frac{(1-P b)}{G s b}}\right]$

Donde:

Gmb: Peso específico bulk de la mezcla asfáltica compactada.

Gse: Peso específico efectivo de los agregados. 
Gb: Peso específico del asfalto.

Gsb: Peso específico bulk de la combinación de agregados.

$\mathrm{Pb}$ : Porcentaje de asfalto.

\%Va: Porcentaje de volumen de vacíos con aire respecto al volumen total de la mezcla.

\%VAM: Porcentaje de vacíos en el agregado mineral en la mezcla compactada.

\%VFA: Porcentaje de vacíos llenos de asfalto.

Con el fin de obtener el módulo dinámico de las mezclas, se realiza el ensayo de tracción indirecta, usando el equipo Nottingham Asphalt Tester-NAT-; este ensayo consiste en aplicar una carga de compresión a diferentes temperaturas y frecuencias, mediante un dispositivo que emite la carga verticalmente en forma de onda, a una probeta de mezcla asfáltica, obteniendo la deformación horizontal, y mediante la relación de Poisson asumida se logra determinar el módulo resiliente de la mezcla. En la Fig. 1 se muestra el equipo empleado.

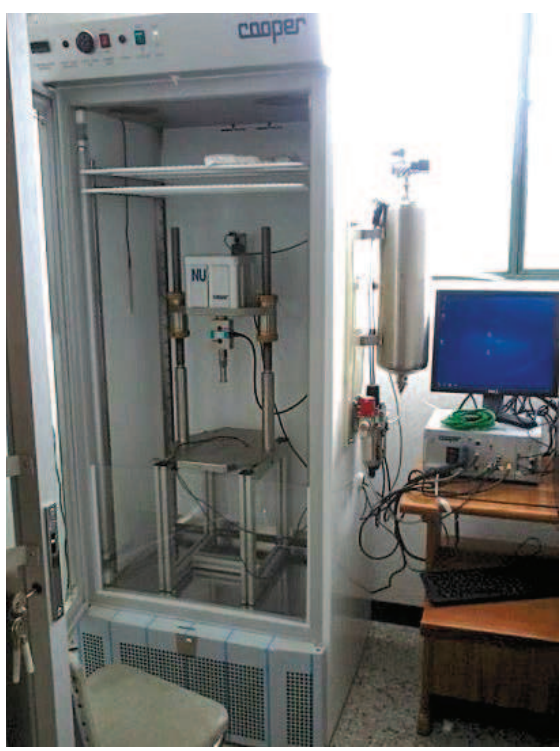

Fig. 1. Equipo NAT -Nottingham Asphalt Tester-. Laboratorio de Pavimentos de la Uptc.

\section{Diseño experimental}

Para el desarrollo de la presente investigación se planteó un diseño factorial de $2^{2}$, mediante el cual se determinó la cantidad de ensayos necesarios; para este caso, el total de experimentos fue de cuatro. En la Fig. 2 se presenta de forma más detallada el diseño experimental planteado. Las variables tenidas en cuenta son, básicamente, el origen de los agregados pétreos y el tipo de mezcla realizada.

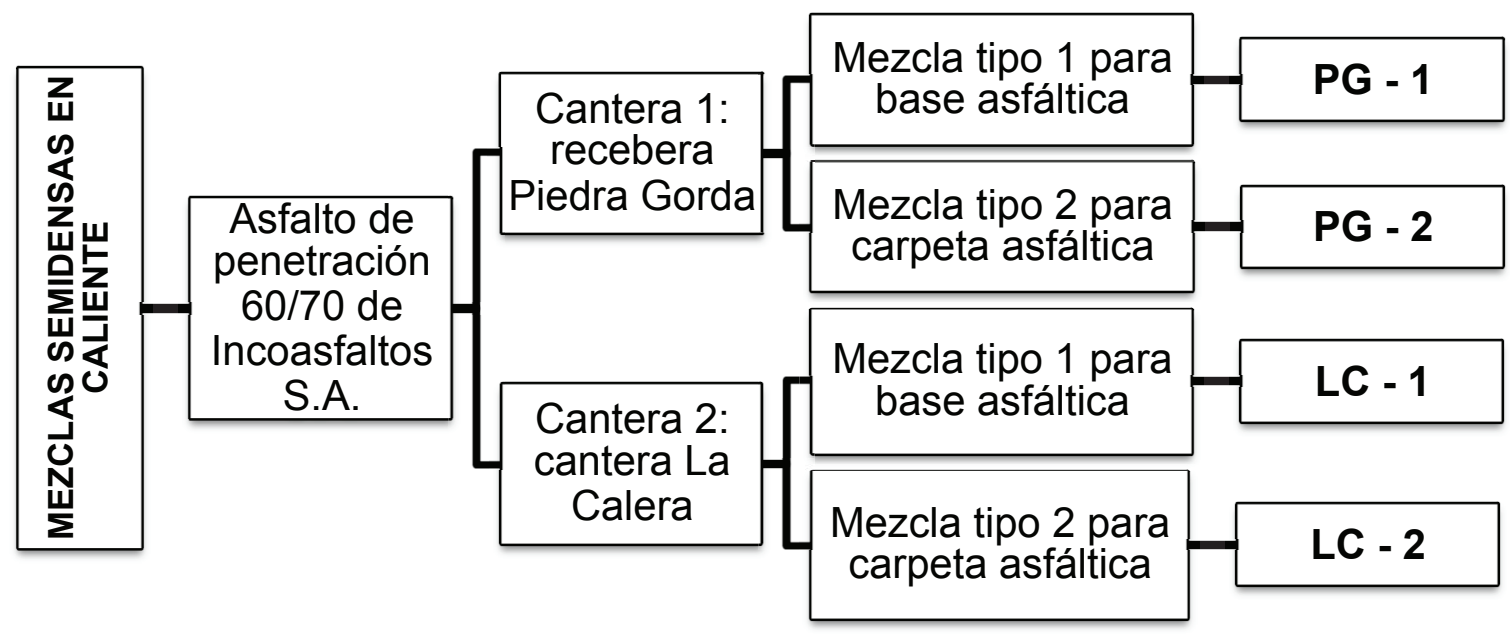

Fig. 2. Variables del diseño experimental para mezclas asfálticas 
Las mezclas se realizaron así: en primer lugar se tomaron los agregados obtenidos de cada una de las dos fuentes de la región de Tunja y se realizó el tipo de mezcla según su uso granulométrico (MSC 1 y MSC 2); una vez obtenido esto se hizo la mezcla con el asfalto de penetración 60/70.

Basados en lo anterior, se realizaron cuatro ensayos Marshall, elaborando 60 briquetas, con el fin de determinar el porcentaje óptimo de asfalto para cada una de las mezclas; además, con estos resultados se desarrolló la metodología Ramcodes, fabricando 12 briquetas en la implementación de esta metodología. Cada una de las mezclas fue elaborada cumpliendo con la franja granulométrica central establecida en la normatividad vigente, cuya gradación es de $47.5 \%$ para agregado pétreo grueso y $52.5 \%$ para agregado pétreo fino. Cabe destacar que el proceso de compactación fue realizado a través del uso del compactador giratorio que se presenta en la Fig. 3.

El ensayo de módulo dinámico se realiza a tres temperaturas diferentes $\left(5{ }^{\circ} \mathrm{C}, 25^{\circ} \mathrm{C}\right.$ y $\left.40{ }^{\circ} \mathrm{C}\right)$, con una frecuencia de 10 hertz [6]; por cada una de las temperaturas, el ensayo se debe realizar dos veces: la primera en su posición inicial, y la segunda con un giro de $90^{\circ}$; antes de realizar el ensayo de tensión indirecta, las briquetas se llevan a la cámara de temperatura del NAT, durante aproximadamente dos horaS, con el fin de que encuentren la temperatura requerida por el ensayo. Para este ensayo se realizaron 12 briquetas en total.

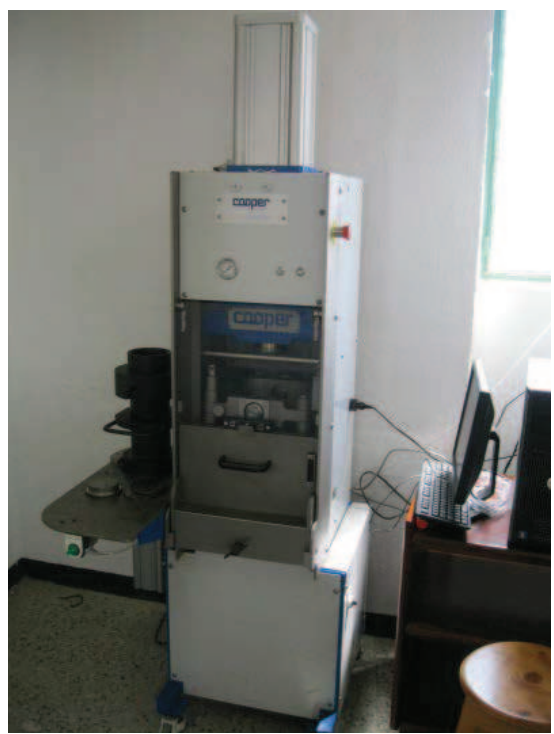

Fig. 3. Compactador giratorio

\section{Resultados}

\section{A. Resultados obtenidos de la imple- mentación de las metodologías Marshall y Ramcodes}

La metodología Ramcodes se basa principalmente en un experimento factorial de dos factores: el peso específico bulk y el porcentaje óptimo de asfalto, en el comportamiento de la mezcla asfáltica. Teniendo en cuenta que esta metodología relaciona parámetros de la metodología Marshall, se espera que los resultados obtenidos en cada mezcla sean similares tanto en sus propiedades volumétricas como mecánicas. En la Tabla 5 se presentan los resultados obtenidos de la implementación de las metodologías Marshalll y Ramcodes. 
TABLA 5

COMPARACIÓN DE RESULTADOS OBTENIDOS POR MEDIO DE LA IMPLEMENTACIÓN DE METODOLOGÍAS MARSHALL Y RAMCODES -RAM-

\begin{tabular}{|c|c|c|c|c|c|c|c|c|}
\hline \multirow{2}{*}{ Parámetro } & \multicolumn{2}{|c|}{ Mezcla PG 1 } & \multicolumn{2}{c|}{ Mezcla PG 2 } & \multicolumn{2}{c|}{ Mezcla LC 1 } & \multicolumn{2}{c|}{ Mezcla LC 2 } \\
\cline { 2 - 9 } & Marshall & RAM & Marshall & RAM & Marshall & RAM & Marshall & RAM \\
\hline \% Pb & 5.9 & 5.7 & 6.2 & 6.4 & 5.78 & 5.43 & 6.99 & 6.63 \\
\hline Gmb (g/cm3) & 2.284 & 2.293 & 2.292 & 2.283 & 2.312 & 2.281 & 2.275 & 2.283 \\
\hline Va (\%) & 5.2 & 5.11 & 3.3 & 4.27 & 5.53 & 5.61 & 4 & 4.24 \\
\hline VAM (\%) & 15.6 & 15 & 15.45 & 16.003 & 15.52 & 14.97 & 15.24 & 15.98 \\
\hline VFA (\%) & 67 & 65.9 & 78 & 73.28 & 64.37 & 62.51 & 73.74 & 73.45 \\
\hline Estabilidad (kg) & 1420 & 3143.45 & 1340 & 1987.68 & 1691.72 & 3976 & 1638.48 & 3220.67 \\
\hline Flujo (mm) & 4.2 & 4.42 & 4.8 & 4.94 & 4.06 & 3.88 & 4.31 & 5.18 \\
\hline
\end{tabular}

En la Fig. 4 se observan los polígonos de vacíos de las cuatro mezclas realizadas con los agregados de las dos canteras; estos se estiman con los resultados de los porcentajes de asfalto y la gravedad bulk, generados con la metodología Ramcodes.

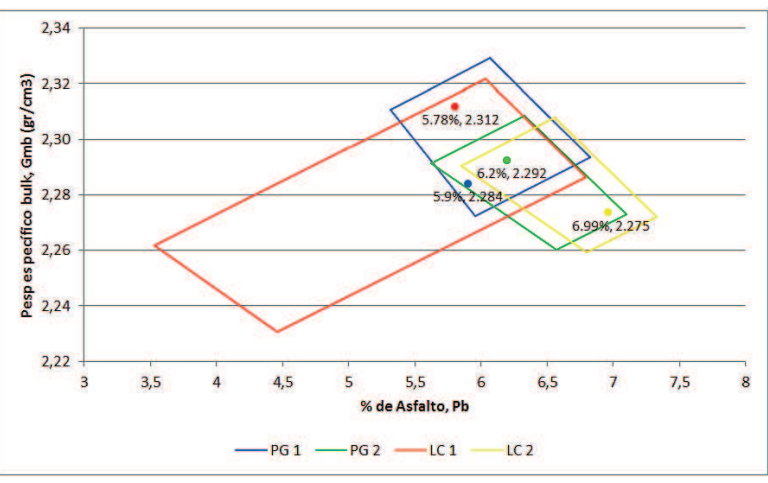

Fig. 4. Polígono de vacío generados con la metodología Ramcodes

\section{B. Resultados obtenidos de las mezclas realizadas con contenido óptimo de asfalto}

Se realizaron 12 briquetas con los resultados de contenido óptimo de asfalto, obtenido a través de la implementación de la metodología Marshall, con el fin de ejecutar el ensayo de tracción indirecta. Los resultados de los ensayos correspondientes a las mezclas tipo 1 y tipo 2 se presentan en las Tablas 6 y 7, respectivamente.

\section{TABLA 6}

RESULTADOS DE LOS ENSAYOS DE LAS MEZCLAS TIPO 1

\begin{tabular}{|c|c|c|c|c|}
\hline \multirow{2}{*}{ Parámetro } & \multicolumn{4}{|c|}{ MSC 1 } \\
\cline { 2 - 5 } & INVÍAS-07 & IDU-05 & PG 1 & LC 1 \\
\hline $\mathbf{\% ~ P b ~}$ & - & - & 5.9 & 5.78 \\
\hline Gmb (g/cm $)$ & - & - & 2.284 & 2.312 \\
\hline Va (\%) & $5-9$ & $4-6$ & 5.2 & 5.53 \\
\hline VAM (\%) & $\geq 14$ & $\geq 14$ & 15.6 & 15.52 \\
\hline VFA (\%) & $65-78$ & $65-75$ & 67 & 64.37 \\
\hline $\begin{array}{c}\text { Estabilidad } \\
(\mathbf{( k g )}\end{array}$ & 750 & 900 & 1420 & 1692 \\
\hline Flujo (mm) & $2-4$ & $2-3.5$ & 4.2 & 4.06 \\
\hline
\end{tabular}

\section{TABLA 7}

RESULTADOS DE LOS ENSAYOS DE LAS MEZCLAS TIPO 2

\begin{tabular}{|c|c|c|c|c|}
\hline \multirow{2}{*}{ Parámetro } & \multicolumn{4}{|c|}{ MSC 2 } \\
\cline { 2 - 5 } & INVÍAS-07 & $\begin{array}{c}\text { IDU- } \\
\mathbf{0 5}\end{array}$ & PG 2 & LC 2 \\
\hline \% Pb & - & - & 6.2 & 6.99 \\
\hline Gmb (g/cm $)^{3}$ & - & - & 2.292 & 2.275 \\
\hline Va (\%) & $5-9$ & $4-6$ & 3.3 & 4.00 \\
\hline VAM (\%) & $\geq 14$ & $\geq 14$ & 15.45 & 15.24 \\
\hline VFA (\%) & $65-78$ & $65-75$ & 78 & 73.74 \\
\hline Estabilidad (kg) & 750 & 900 & 1340 & 1638 \\
\hline Flujo (mm) & $2-4$ & $2-3.5$ & 4.8 & 4.3 \\
\hline
\end{tabular}


Según lo que se observa en las Tablas 6 y 7, se puede inferir que para las mezcla PG 2, el porcentaje de asfalto requerido es un poco mayor que el que se requiere para la mezcla PG 1, debido a que la superficie específica del agregado que se necesita para el recubrimiento total es mayor, dada la granulometría misma de la mezcla. Se determina que los valores óptimos para los parámetros del \%Va, \%VAM y \%VFA cumplen con las especificaciones del INVÍAS-07 e IDU-05. En cuanto a la estabilidad, el material no presenta valores menores a los de la norma y se tiene que la resistencia a la compresión es mayor en las mezcla PG 1.

En el caso de las mezclas realizadas con el agregado pétreo de la cantera La Calera, tanto para base asfáltica como para carpeta asfáltica, se cumple con los parámetros de vacíos con aire (\%Va), vacíos del agregado mineral (\%VAM) y vacíos llenos de asfalto (\%VFA). De igual manera, el valor de estabilidad obtenido no presenta valores menores a los de la norma, y se tiene que presenta alta resistencia a la compresión. Por otra parte, la estabilidad supera los valores exigidos por la normatividad vigente, es decir, se alcanzan valores superiores a los $1300 \mathrm{~kg}$., logrando que todas las mezclas realizadas cumplan con lo establecido por el INVÍAS-07 y por el IDU-05.

\section{Resultados del ensayo de tracción indirecta}

Las briquetas para el ensayo de módulo dinámico fueron compactadas con el compactador giratorio; mediante éste se logran indicar algunas de las características volumétricas y mecánicas de cada una de las mezclas realizadas, además, se puede obtener una comprobación de los datos iniciales ingresados.

Los resultados que se obtienen con el ensayo en el NAT (Nottingham Asphalt Tester), para las mezclas semidensas en caliente, realizadas con los resultados del porcentaje óptimo de asfalto de la metodología Marshall, se muestran en el Tabla 8.

TABla 8

ReSUlTADOS DE MÓDULOS DINÁMICOS

\begin{tabular}{|c|c|c|c|c|c|}
\hline \multirow{4}{*}{$\begin{array}{c}\text { Temperatura } \\
\left({ }^{\circ} \mathbf{C}\right)\end{array}$} & Mediciones & \multicolumn{2}{|c}{$\begin{array}{c}\text { Módulo dinámico de las } \\
\text { mezclas con el agregado de } \\
\text { Piedra Gorda (MPa) }\end{array}$} & \multicolumn{2}{c|}{$\begin{array}{c}\text { Módulo dinámico de las } \\
\text { mezclas con el agregado La } \\
\text { Calera (MPa) }\end{array}$} \\
\cline { 3 - 6 } & & MSC 1 & MSC 2 & MSC 1 & MSC 2 \\
\hline \multirow{4}{*}{5} & 1 & 13552 & 12308 & 11450 & 10528 \\
\cline { 2 - 6 } & 2 & 13846 & 11972 & 11303 & 10912 \\
\cline { 2 - 6 } & Promedio & $\mathbf{1 3 6 9 9}$ & $\mathbf{1 2 1 4 0}$ & $\mathbf{1 1 3 7 6}$ & $\mathbf{1 0 7 2 0}$ \\
\hline \multirow{4}{*}{$\mathbf{2 5}$} & 1 & 4002 & 4050 & 1847 & 2683 \\
\cline { 2 - 6 } & 2 & 3768 & 3887 & 1721 & 2429 \\
\hline \multirow{4}{*}{40} & Promedio & $\mathbf{3 8 8 5}$ & $\mathbf{3 9 6 9}$ & $\mathbf{1 7 8 4}$ & $\mathbf{2 5 5 6}$ \\
\cline { 2 - 6 } & 1 & 1390 & 822 & 481 & 583 \\
\cline { 2 - 6 } & 2 & 1273 & 802 & 447 & 493 \\
\hline & Promedio & $\mathbf{1 3 3 1}$ & $\mathbf{8 1 2}$ & $\mathbf{4 6 4}$ & $\mathbf{5 3 8}$ \\
\hline
\end{tabular}


De acuerdo con lo que se presenta en la Tabla 8, las briquetas elaboradas con los agregados de la recebera Piedra Gorda presentan valores mayores en los módulos para las tres temperaturas del ensayo, en comparación con las briquetas falladas con los agregados de la cantera La Calera.

Resulta característico que esta situación se haya presentado, debido a que los agregados de la Recebera Piedra Gorda presentaban una forma y textura que hacía que las briquetas presentaran mayor altura y que, a simple vista, se observaba que la contextura plana y alargada de los agregados gruesos la convertían en un material propenso a la fractura al momento de la compactación. A su vez, la poca adherencia que tenía el agregado con el asfalto a la hora de realizar el mezclado manual, hacía que algunas partículas, principalmente las más grandes, no se recubrieran completamente con el ligante bituminoso. Estos fenómenos que se presentaban, tanto para el agregado como para la mezcla, hacían pensar a los investigadores que la mezclas MSC 1 y MSC 2 con este agregado no servirían para su uso en vías con volúmenes de tránsito altos; sin embargo, la mezcla semidensa en caliente con granulometría tipo 1 sería una buena alternativa para cualquiera de las capas asfálticas (carpeta o base).

Las mezclas elaboradas con el agregado de la cantera La Calera presentaron unos valores de módulos dinámicos menores; sin embargo, y teniendo en cuenta que este material granular presentaba una contextura más uniforme y con una adherencia adecuada con el asfalto, se podría establecer que la MSC 2 sería una buena alternativa para cualquiera de las capas asfálticas (carpeta o base).

Teniendo en cuenta todos los resultados, se observa que el comportamiento de los módulos dinámicos es inversamente proporcional a la temperatura del ensayo, es decir, que a menor temperatura $\left(5^{\circ} \mathrm{C}\right)$ el valor en el módulo, medido en $\mathrm{MPa}$, es mayor que con ensayos a temperaturas superiores $\left(25^{\circ} \mathrm{C}\right.$ y 40 $\left.{ }^{\circ} \mathrm{C}\right)$.

En la Fig. 5 se presentan las curvas maestras de comportamiento de los módulos dinámicos para las mezclas semidensas en caliente; son construidas, principalmente, por la superposición del tiempo de carga (frecuencia) y la temperatura. La finalidad de dichas curvas es describir la relación que tiene el material con el tiempo, con el fin de observar parámetros como la velocidad de los vehículos en función de la resistencia de la mezcla asfáltica y la temperatura del lugar; de igual manera, se puede observar el comportamiento que tienen las mezclas semidensas a las temperaturas de ensayo. Teniendo en cuenta que la Temperatura Media Anual Ponderada (TMAP) de la región de Tunja es de 13 ${ }^{\circ} \mathrm{C}$, las mezclas elaboradas con el agregado de la recebera Piedra Gorda presentan módulos de 8000 MPa para las dos granulometrías; mientras que las mezclas con el agregado de la cantera La Calera presentan un valor de $5000 \mathrm{MPa}$ para el caso de las MSC 1, y de 6000 MPa para el caso de las MSC 2.

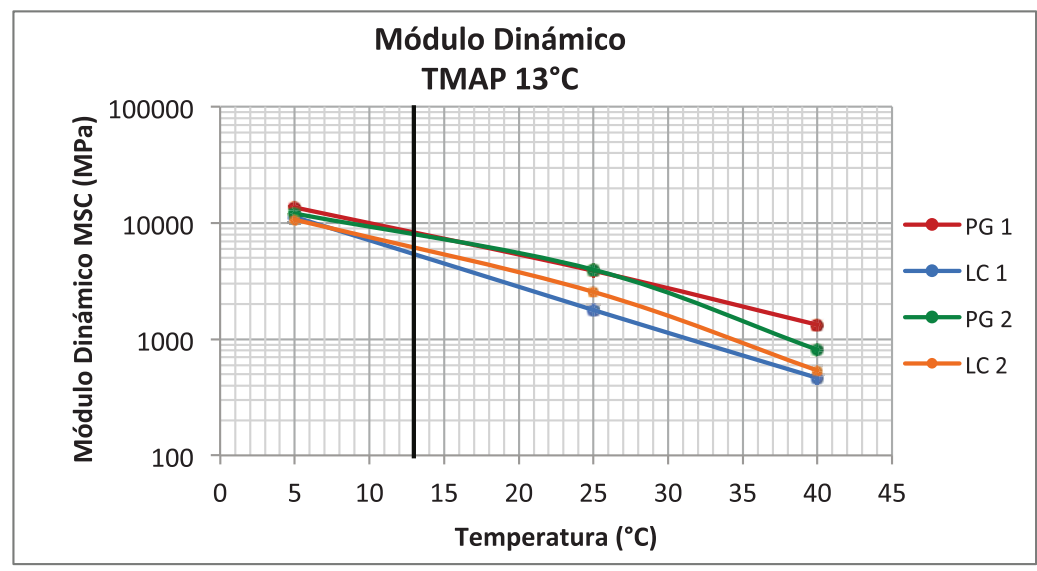

Fig. 5. Curvas maestras de comportamiento de los módulos dinámicos 
El valor del módulo dinámico de las mezclas asfálticas determinado a $20^{\circ} \mathrm{C}$ permite deducir que las mezclas realizadas con el agregado de la Recebera Piedra Gorda presentan valores superiores de $5000 \mathrm{MPa}$, mientras que las mezclas realizadas con el agregado de la cantera La Calera presenta valores superiores a $2800 \mathrm{MPa}$.

\section{Conclusiones}

Los resultados obtenidos en la caracterización del agregado pétreo indican que el material usado en esta investigación es apto para realizar las mezclas asfálticas semidensas, debido a que cumplen por completo los requerimientos estipulados por el INVÍAS-07 y el IDU-05.

El asfalto de penetración 60/70 procedente de IncoAsfaltos S.A. cumple con los criterios establecidos en las normas del INVÍAS-07 y del IDU-05, por lo que es apto para la construcción de vías de tránsito medio.

Con la implementación de la metodología Marshall se determinaron las fórmulas de trabajo de cada una de las mezclas elaboradas, así: para la mezcla PG $1,5.9 \%$ de asfalto y $94.1 \%$ de agregado pétreo; para PG 2, 6.2\% de asfalto y $93.8 \%$ de agregado pétreo; para LC 1, 5.78\% de asfalto y $88.44 \%$ de agregado pétreo, y para LC $2,6.99 \%$ de asfalto y $93.01 \%$ de agregado pétreo.

Los valores de estabilidad obtenidos superan considerablemente lo requerido por las normas INVÍAS-07 e IDU-05, ya que para todas las mezclas este valor supera los $1340 \mathrm{~kg}$. En cuanto al parámetro de flujo, ninguno de los valores obtenidos se encuentra en el rango de las especificaciones, pero no están muy alejados de la especificación.

El uso del compactador giratorio permite confirmar los valores de los parámetros de algunas propiedades volumétricas y físicas de las briquetas elaboradas, ya que su software requiere parámetros que fueron obtenidos en la metodología desarrollada.

El ensayo de tensión indirecta, realizado en el equipo NAT (Nottingham Asphalt Tester), determina que las mezclas realizadas con agregado pétreo de la recebera Piedra Gorda presentan mayores valores de módulo dinámico, en comparación con las mezclas realizadas con agregado pétreo de la cantera La Calera; de igual manera, las mezclas tipo 1 , realizadas para bases asfálticas, presentan mayor resistencia a la deformación por tensión indirecta.

Se recomienda continuar con investigaciones acerca de la caracterización y diseño de mezclas semidensas, usando agregado pétreo de diferentes lugares del departamento de Boyacá, con el fin de establecer características de nuevos materiales que sean aptos para su implementación en vías regionales y nacionales.

\section{Agradecimientos}

Los autores expresan sus agradecimientos a la Escuela de Transporte y Vías de la Facultad de Ingeniería de la Universidad Pedagógica y Tecnológica de Colombia por el aporte brindado al desarrollo de este proyecto de investigación. Igualmente, al Ingeniero Manuel Sierra, coordinador del Laboratorio de Suelos y Pavimentos de la Uptc, por su colaboración en el desarrollo de la investigación.

\section{REFERENCIAS}

[1] Ministerio de Transporte. Oficina de Planeación. Transporte en cifras. Estadísticas de la red vial de Colombia. Bogotá, D.C. 2012.

[2] The Global Competitiveness Report 20112012. World Economic Forum. Estados Unidos. 2012. 
[3] Instituto Nacional de Vías -INVÍAS-. Especificaciones generales de construcción de carreteras. Artículo 450-07. Bogotá, 2007.

[4] Instituto de Desarrollo Urbano -IDU-. Especificaciones técnicas generales de materiales y construcción para proyectos de infraestructura vial y del espacio público en Bogotá, D.C. Capítulo 5. Bogotá, 2005.
[5] F. Sánchez, P. Garnica, J. Gómez and N. Pérez. Ramcodes: metodología racional para el análisis de densificación y resistencia de geomateriales compactados. Publicación Técnica N. ${ }^{\circ} 200$. Sanfandila. México, 2002. ISSN 0188-7297.

[6] Instituto Nacional de Vías -INVÍASNormas generales de construcción de carreteras. Norma INV E-749-07. Bogotá, 2007. 